\title{
Discrete element modelling of large scale particle systems-I: exact scaling laws
}

\author{
Y. T. Feng - D. R. J. Owen
}

Received: 28 February 2014 / Revised: 21 March 2014 / Accepted: 27 March 2014 / Published online: 15 April 2014

(C) Springer International Publishing Switzerland 2014

\begin{abstract}
The discrete element method has emerged as a powerful predictive tool for the numerical modelling of many scientific and engineering problems involving discrete and discontinuous phenomena. There are nevertheless computational challenges to resolve before industrial scale applications can be effectively simulated. This multi-part paper aims to address some of the theoretical and computational issues central to achieving this goal. In the first part of this paper, a simple but generic theoretical framework is established for the development of a comprehensive set of scaling conditions, under which a scaled discrete element model can exactly reproduce the mechanical behaviour of a physical model. In particular, three basic physical quantities and their scale factors can be freely chosen. A special selection leads to a unique set of scale factors governing an exact scaling, which also gives rise to the requirement that all the interaction laws employed in a scaled model be scale-invariant. The subsequent examination reveals that most commonly used interaction laws, if all material (mechanical and physical) properties are treated as constant, do not possess such a feature and therefore cannot be directly employed in a scaled model. The problem can be solved by treating the scaled particles as pseudo-particles and by properly scaling the interaction laws. The resulting scaled interaction laws become scale-invariant and thus can be used in a scaled model.
\end{abstract}

Keywords Discrete element method - Scale factor - Exact scaling $\cdot$ Interaction law $\cdot$ Scale invariance

Y. T. Feng $(\varangle)$ · D. R. J. Owen

Zienkiewicz Centre for Computational Engineering,

College of Engineering, Swansea University,

Swansea SA2 8PP, UK

e-mail: y.feng@swansea.ac.uk

\section{Introduction}

The last ten years have witnessed rapidly increasing research interest in the development of different particle based methods for a wide range of applications. As one of the dominant particle-based methods, the discrete element method (DEM) has become firmly established as the most powerful numerical technique to model many scientific and engineering problems involving particle/particulate phenomena since it was originated in the 1970s [1]. The DEM, in its classic form, represents individual (disjoint) physical particles as (rigid) elements which interact only along their interfaces. Although significant advances have been made over the last two decades in many aspects of discrete element modelling, some fundamental and practical issues remain to be properly addressed. Among the computational challenges to be tackled, the ability of the DEM to simulate industrial- or macro-scaled problems within a realistic computer time scale is probably the most pressing issue from the application point of view.

The problem scale of a physical or discrete element model may be represented by introducing a scale number, $\mathrm{S}_{n}$, defined as

$S_{n}=D / R$

where $D$ and $R$ are the characteristic dimension of the problem concerned and the characteristic size of discrete elements used respectively. A scale classification shown in Table 1 may be adopted.

(Micro-scale) problems with less than several million particles have been routinely modelled by DEM. However, real industrial applications involve, at least, billions of particles and such applications, classified as macro-scale problems, cannot be effectively modelled yet due to the limitation of computer resources currently available. There are sev- 
Table 1 Scale classification of particle systems

\begin{tabular}{lll}
\hline Scale & Scale number $S_{n}$ & $\begin{array}{l}\text { Typical number } \\
\text { of particles }\end{array}$ \\
\hline Micro-scale & $<10^{2}$ & $<10^{6}$ \\
Meso-scale & $10^{2}-10^{3}$ & $10^{6}-10^{9}$ \\
Macro-scale (industrial) & $>10^{3}$ & $>10^{9}$ \\
\hline
\end{tabular}

eral possible solutions, including parallelisation, continuum approximations and coarse-graining approaches.

Use of parallel computing platforms to speed up DEM simulations [2] may be the most straightforward solution. However, apart from having access to the most powerful parallel computing facilities in the world, the required computational costs for simulating a realistic industrial problem are still extremely excessive. In the foreseeable future, even the fast growth of computing power is very unlikely to meet the ever increasing practical needs.

Continuum approximations offer a different approach whereby the discrete particle systems are represented as a continuum medium by applying various homogenisation or averaging techniques [3]. This approach aims to establish a set of balance equations with unique and time-varying constitutive models for the problem concerned, which then can be solved by using standard continuous based numerical techniques such as finite element methods to significantly reduce the computational costs. Although this is a very promising approach, and particularly when viewed within a multi-scale modelling framework, it may not be able to capture all essential physical characteristics of many granular systems of an inherent discrete and diversity nature.

On the other hand, coarse graining techniques, a term borrowed from Molecular Dynamics, have recently gained some attention as a possible approach to tackle macroscale problems, in which particles are artificially enlarged or (up-)scaled in DEM models. As a result, the number of discrete elements required can be significantly reduced and the original problems can be solved within a reasonable time scale. Several versions of coarse-graining are available [4-6], but they are generally derived in an ad-hoc manner, and some questions remain to be answered before the techniques can be fully employed.

This multi-part paper aims to address some of the theoretical and computational issues central to the effective modelling of macro-scale industrial problems. The first part of the paper attempts to answer the question of under what conditions a scaled discrete element model can exactly represent the original physical problem. The second part reviews existing coarse graining techniques for discrete element modelling of particle systems so as to propose a general and systemic development framework. The third part develops a new methodology to estimate the solution accuracy.
In our previous work [7], a set of scaling laws for upscaling of discrete element models are proposed in terms of three similarity principles, namely geometrical, mechanical and temporal. The current work is an extension and further enhancement of the approach in that a much simpler and more generic theoretical framework is established for the development of a comprehensive set of scaling laws governing the exact scaling of a discrete element model. This will lay the foundation for the developments in the subsequent parts of the paper.

\section{Scaled model: exact scaling conditions}

\subsection{Physical and scaled models}

Consider a general particle system (referred to as the physical model in what follows) and a possibly scaled discrete element model (referred to as the scaled model). The physical model is assumed to comprise particles that have various size distributions and are (randomly) packed in a domain. The particles may be subject to the action of external forces (applied and/or gravitational), or driven by other phases, such as fluid, electrical or magnetic fields, or by prescribed displacement and/or velocity on the domain boundaries. The corresponding discrete element model, assumed to have been scaled, is a geometrically exact representation of the physical problem, i.e. both models have the same particle number and particle packing configuration, and the particle sizes and the domains in the two models are different only by a constant (spatial) scale factor $h$.

The scaled model is also assumed to be subjected to the same loading/boundary conditions as in the physical model. In what follows, the overbar ${ }^{-}$will be used to denote a quantity associated with the scaled model. Note that the following discussions are based on a general 3D setting. The same conclusions can be readily obtained by treating a $2 \mathrm{D}$ case as a special 3D case. We further assume that particles are spherical, but the conclusions to be drawn are generally also valid for other shaped particles.

Let $R$ and $\bar{R}$ be the radii of an arbitrary particle in both the physical and scaled models respectively, and $D$ and $\bar{D}$ denote the characteristic lengths of the domains in both models. Then

$\bar{R}=h R ; \quad \bar{D}=h D ; \quad \bar{S}_{n}=S_{n}$

i.e. the scale number $S_{n}$ remains the same for both models. Therefore, the scaled model satisfies the principle of geometric similarity as stated in [7].

\subsection{Particle strain, stress and interaction laws}

A key issue in the modelling of particle systems is to correctly model the interaction forces between a pair of contacting particles. The interaction laws that describe the forces 


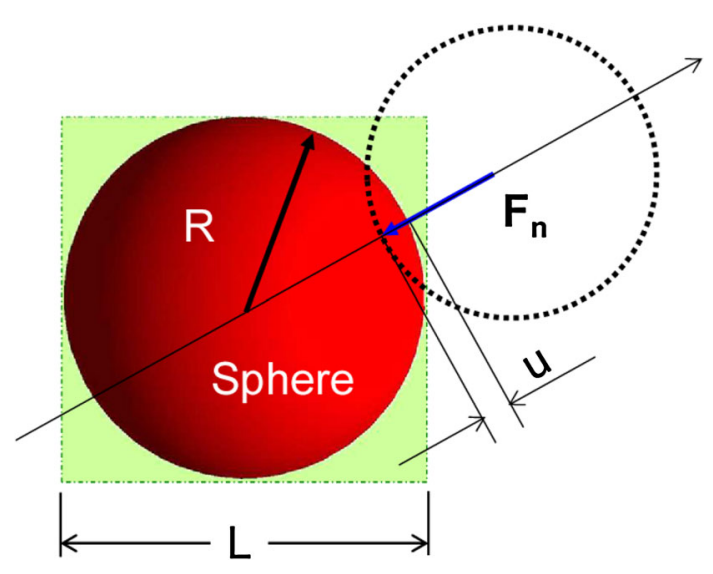

Fig. 1 Representative geometrical features of a sphere

between a pair of particles can be generally expressed in force-displacement format as:

$\mathbf{F}=\mathbf{F}(\mathbf{u}, R, \zeta)$

where $\mathbf{F}$ is the contact force (vector), either in the normal or tangential direction; $R$ represents the intrinsic particle lengthscale; $u$ is the overlap/separation/sliding distance of the two contacting particles whose initial value $u_{0}$ is chosen such that the corresponding force is zero, i.e. $F\left(\mathbf{u}_{0}, R, 0\right)=0$; and $\zeta$ represents some internal parameters or state variables, if they exist. For instance, $\zeta$ may be the accumulated 'total' sliding distance in the 'plasticity theory' based Columbic friction model $[8,9]$, or the maximum pressure experienced in a history pressure dependent cohesive force model. To facilitate the subsequent discussion and to focus on key issues, $\zeta$ is ignored.

Introduce the representative length $L$, area $A$ and volume $V$ of a particle (for a sphere of radius $R, L=2 R ; A=$ $L^{2} ; \quad V=A \times L=L^{3}$. See Fig. 1) and also define the nominal particle (contact) strain and stress respectively as

$\varepsilon=u / L ; \quad \sigma(u, R)=F(u, R) / A$

Then the interaction law can be equivalently expressed in the particle stress-strain format,

$\sigma=\sigma(\varepsilon, R)$

which is the constitutive model in the classic continuum mechanics sense. The above particle strain and stress are defined for each contact with which a given particle is involved. Note, however, that this strain and stress definition is a much simplified treatment of the general (averaged) particle stress and strain of a particle assembly. See [10], for instance, for the topic.

Further define a particle strain (contact) energy function, $E_{S}(u, R)$, that is associated with the interaction force $F(u, R)$ as
$E_{s}(u, R)=\int_{0}^{u} F(u, R) d u$

Similarly, the particle strain (contact) energy density function can be defined as

$e_{s}(R, \varepsilon)=\frac{E(u, R)}{V}=\int_{0}^{\varepsilon} \sigma(\varepsilon, R) d \varepsilon$

while for a multi-contact case,

$e_{S}(R, \varepsilon)=\sum_{i} \int_{0}^{\varepsilon_{i}} \sigma_{i}\left(\varepsilon_{i}, R\right) d \varepsilon$

where the subscript $i$ is used to denote a quantity for the $i$-th contact with which the particle is involved.

In addition, two more energy densities can be defined for each particle: the kinetic energy density: $e_{k}=m v^{2} / 2 \mathrm{~V}=$ $\rho v^{2} / 2$ where $m, V$ and $v$ are the mass, volume and velocity of the particle respectively; and the total energy density: $e=$ $e_{s}+e_{k}$.

\subsection{Exact scaling conditions}

In what follows, a complete set of scaling conditions under which the scaled model can exactly represent the behavior of the original physical model will be established. In our previous work [7], the conditions are expressed in terms of the three similarity principles, whereas the conditions here are derived from the governing equations in a straightforward manner, based on simple unit conversions of all the physical quantities involved in both physical and scaled models. These conditions must be satisfied to ensure that the results obtained in the scaled model can be exactly scaled back to obtain the results for the physical model and vice versa, and therefore define a set of exact scaling laws.

\subsubsection{Governing equations}

The mechanical motion of the particle system is fully governed by Newton's second law. The governing equation for an arbitrary particle can be generally expressed as:

$m \ddot{u}(t)+F_{d}(t)+F_{\text {int }}(t)=F_{\text {ext }}(t)$

where $m$ is the mass of the particle; $F_{d}$ is the damping force accounting for any energy dissipation in the system; $F_{i n t}$ is the resultant of all the interaction forces from other particles or other phases defined by interaction laws; $\mathbf{F}_{\text {ext }}$ is the (resultant) external force applied if any; and $\ddot{u}$ is the acceleration, where $u$ can be viewed as the displacement/position. The same equation can be written for the particle in the scaled system as: 
$\bar{m} \ddot{\vec{u}}(t)+\bar{F}_{d}(t)+\bar{F}_{i n t}(t)=\bar{F}_{\text {ext }}(t)$

The exact scaling laws establish the conditions required to guarantee the equivalence between the physical system and the scaled model, i.e. the two sets of Eqs. (8) and (9) are mathematically equivalent, or simply differ only by a constant factor.

In the classic dimensional analysis [11,12], a set of nondimensional quantities are sought from which a set of scaling laws can be identified. Although some insightful nondimensional parameters may emerge from this approach, the procedure may not be straightforward, and particularly, different sets of non-dimensional parameters may be derived for different applications and in different ways. The same comments also apply to some earlier work on the development of scaling laws for particle systems [13].

Our previous work [7] takes a relatively simple approach, in which a set of scaling laws is identified by ensuring that all the corresponding forces involved in both models are proportional:

$\frac{\bar{m} \ddot{\bar{u}}}{m \ddot{u}}=\frac{\bar{F}_{d}}{F_{d}}=\frac{\bar{F}_{\text {int }}}{F_{\text {int }}}=\frac{\bar{F}_{\text {ext }}}{F_{\text {ext }}}=\lambda \quad$ (constant)

A more straightforward approach is adopted in this work, which aims to directly establish the unit conversion or scale factors of individual physical quantities involved between the physical and scaled models. Let $q$ be an arbitrary physical quantity in the system. A scale factor $\lambda_{q}$ for $q$ is solved such that

$\bar{q}=\lambda_{q} q$

The entire set of scaling laws will be defined when the scale factors for all the physical quantities are determined.

\subsubsection{Independent quantities}

Physical quantities are not all mutually independent but interdependent. There are only a few independent or basic quantities for a physical system, from which all other quantities can be derived.

The scaled computational model provides an opportunity to choose a different set of basic quantities from the conventional SI system. Furthermore, two different sets of the fundamental units can also be assigned to the basic quantities for both physical and scaled models. As a result, other physical quantities can be expressed in terms of the chosen basic quantities and of their respective units in the two models. The exact scaling laws are to determine the unit conversion or scale factors for all the relevant physical quantities between the two models.

It is well known that there are three basic quantities for mechanical systems without considering other physical phases, such as thermal, electrical and magnetic, in the SI sys- tems: Length [L], Mass [M] and Time [T] (where [.] denotes the dimension of a physical quantity, following the common convention), but other selections of three basic quantities are possible. After the three basic quantities are chosen, their corresponding conversion or scale factors between the physical and the scaled models can be arbitrarily set in principle. The other quantities are dependent on the basic quantities, and their conversion or scale factors will be automatically fixed when the three basic scale factors are defined through multiplicative conversion factors.

It is noted that there are different possible selections of the basic quantities and the corresponding scale factors, each leading to a different, but equivalent set of scale factors and thus different scaling laws.

In our work, mass density $[\rho]$ replaces mass as a basic quantity, but length and time remain as the other two basic quantities. The corresponding scale factors for these three quantities, $\lambda_{L}, \lambda_{M}, \lambda_{\rho}$ are specifically chosen as:

$\lambda_{L}=h ; \quad \lambda_{M}=h ; \quad \lambda_{\rho}=1$

i.e. the length scale factor is the same as the (spatial) scale factor $h$ as expected; the temporal (or time) scale is the same as the spatial factor; while the density is kept the same for both systems. The advantages of such a special choice of the basic scale factors will become apparent later.

\subsubsection{Derived quantities and scale factors}

After the three basic quantities and the corresponding scale factors are defined, it is a simple exercise to work out the scale factors for all the derived quantities. Table 2 lists commonly used physical quantities in particle systems, their scale factors and dimensions in terms of the three chosen basic quantities. The quantities are also grouped, in an ad-hoc manner, into several categories including: basic, geometrical, kinematic, dynamic and material. The list is, however, by no means exhaustive, and additional quantities can be readily added if needed. Except for angle and strain, other nondimensional quantities are not included in the table because they remain dimensionless and the scale factors are equal to one.

The best way to work out the scale factor for a quantity is to express its dimension in terms of the basic quantities and then to convert the dimensions to the scale factors. For instance, for mass, using $M=\rho V$ gives

$[M]=[\rho][L]^{3} ; \quad \lambda_{M}=\lambda_{\rho} \lambda_{L}^{3}=h^{3}$

and for force, using $F=m a$ gives

$[F]=[M][L][T]^{-2}=[\rho][L]^{4}[T]^{-2} ; \quad \lambda_{F}=\lambda_{\rho} \lambda_{L}^{4} \lambda_{T}^{-2}=h^{2}$ 
while for Young's modulus $E$, consider the relationship between the axial force $F$ and the axial displacement $u$ of a 1D bar with Young's modulus $E$, cross-section $A$ and length $L$ :

$F=\frac{E A}{L} u$

which leads to

$[E]=[\rho][L]^{2}[T]^{-2} ; \quad \lambda_{E}=\lambda_{\rho} \lambda_{L}^{2} \lambda_{T}^{-2}=1$

Note that Table 2 is compiled for 3D cases. A similar table can be obtained for 2D cases by the fact that the characteristic length, area and volume of a particle can be collectively described for both 2D and 3D cases as

$L=2 R ; \quad A=L^{n_{d}-1} ; \quad V=A L$

where $n_{d}$ is the number of spatial dimensions concerned.

Table 2 shows that some quantities have the scale factors equal to one. These are the direct consequence of the particular choice of the three basic quantities and their scale factors and lead to some desirable results. In addition, a few remarks on some subtle issues deserve to be made.

Remark 1 (Equivalence of particle energy density evolution) Utilising the particle kinetic and strain energy (volume) densities, $e_{k}$ and $e_{s}$, defined for a particle in Sect. 2.2, and further defining $e_{d}=\int F_{d} d u / V$ (where $u$ is the displacement, and $\mathrm{V}$ is the volume of the particle) as the total energy (volume) density dissipated, and $w=\int F_{\text {ext }} d u / V$ as the total work done per volume by all the external forces acting on the particle, the governing Eq. (8) for a particle can be re-written in an equivalent, energy rate balance format as

$\dot{e_{k}}+\dot{e_{d}}+\dot{e_{s}}=\dot{w}$

The unity of the scale factors for $e_{k}$ and $e_{s}$ from the table (and obviously the same for $e_{d}$ and $w$ ) indicates that the energy densities per particle are identical between the physical and scaled models, ensuring an exact scaling between the two models.

Remark 2 (Partial scaling for interaction laws) The fundamental feature that governs the the exact scaling of interaction laws is dictated by the conclusion that the scale factors for particle strain and stress are unity, as shown in the table.

Table 2 also lists some selected physical quantities that may be involved in most particle systems. Except for mass or mass density, the other quantities are mainly used in particle interaction laws. This gives rise to two possible approaches to imposing the exact scaling conditions on interaction laws. In the first approach, the required scale factors are applied consistently across to all the quantities involved, and this seems to a desirable approach. Nevertheless, in practice, most of material and model parameters in an interaction law are
Table 2 Selected physical quantities, symbols, dimensions and scale factors

\begin{tabular}{|c|c|c|c|c|}
\hline Quantity name & Symbol & Dimensions & $\begin{array}{l}\text { Scale } \\
\text { factor }(\lambda)\end{array}$ & Category \\
\hline Length & $\mathrm{L}$ & {$[L]$} & $h$ & Basic \\
\hline Time & $\mathrm{T}$ & {$[T]$} & $h$ & Basic \\
\hline Density & $\rho$ & {$[\rho]$} & 1 & Basic \\
\hline (Spatial) Position & $X$ & {$[L]$} & $h$ & Geometrical \\
\hline $\begin{array}{l}\text { Angular position, } \\
\text { angle of } \\
\text { rotation }\end{array}$ & $\theta$ & {$[1]$} & 1 & Geometrical \\
\hline Area & A & {$[L]^{2}$} & $h^{2}$ & Geometrical \\
\hline Volume & $\mathrm{V}$ & {$[L]^{2}$} & $h^{3}$ & Geometrical \\
\hline $\begin{array}{c}\text { Displacement, } \\
\text { Overlap etc }\end{array}$ & $\mathrm{u}$ & {$[L]$} & $h$ & Kinematic \\
\hline Velocity & $\mathrm{v}$ & {$[L][T]^{-1}$} & 1 & Kinematic \\
\hline Acceleration & $\mathrm{a}$ & {$[L][T]^{-2}$} & $h^{-1}$ & Kinematic \\
\hline Angular velocity & $\dot{\theta}$ & {$[T]^{-1}$} & $h^{-1}$ & Kinematic \\
\hline $\begin{array}{l}\text { Angular } \\
\text { acceleration }\end{array}$ & $\ddot{\theta}$ & {$[T]^{-2}$} & $h^{-2}$ & Kinematic \\
\hline $\begin{array}{l}\text { (Natural) } \\
\text { Frequency }\end{array}$ & $\omega$ & {$[T]^{-1}$} & $h^{-1}$ & Dynamic \\
\hline Force & $\mathrm{F}$ & {$[\rho][L]^{4}[T]^{-2}$} & $h^{2}$ & Mechanical \\
\hline Moment & Mo & {$[\rho][L]^{5}[T]^{-2}$} & $h^{3}$ & Mechanical \\
\hline Stiffness & $k$ & {$[\rho][L]^{3}[T]^{-2}$} & $h$ & Mechanical \\
\hline Strain & $\epsilon$ & {$[1]$} & 1 & Mechanical \\
\hline Stress & $\sigma$ & {$[\rho][L]^{2}[T]^{-2}$} & 1 & Mechanical \\
\hline $\begin{array}{l}\text { Kinetic energy } \\
\text { (volume) } \\
\text { density }\end{array}$ & $e_{k}$ & {$[\rho][L]^{2}[T]^{-2}$} & 1 & Mechanical \\
\hline $\begin{array}{l}\text { Strain energy } \\
\text { (volume) } \\
\text { density }\end{array}$ & $e_{s}$ & {$[\rho][L]^{2}[T]^{-2}$} & 1 & Mechanical \\
\hline $\begin{array}{l}\text { Energy (volume) } \\
\text { density }\end{array}$ & $e$ & {$[\rho][L]^{2}[T]^{-2}$} & 1 & Mechanical \\
\hline Mass & M & {$[\rho][L]^{3}$} & $h^{3}$ & Material \\
\hline Young's modulus & $\mathrm{E}$ & {$[\rho][L]^{2}[T]^{-2}$} & 1 & Material \\
\hline Surface tension & $\kappa$ & {$[\rho][L]^{3}[T]^{-2}$} & $h$ & Material \\
\hline $\begin{array}{l}\text { Surface energy } \\
\text { density }\end{array}$ & $\gamma$ & {$[\rho][L]^{3}[T]^{-2}$} & $h$ & Material \\
\hline $\begin{array}{l}\text { Viscosity } \\
\text { (dynamic) }\end{array}$ & $\eta$ & {$[\rho][L]^{2}[T]^{-1}$} & $h$ & Material \\
\hline $\begin{array}{l}\text { Viscosity } \\
\text { (kinematic) }\end{array}$ & $v$ & {$[L]^{2}[T]^{-1}$} & $h$ & Material \\
\hline
\end{tabular}

often viewed, either conveniently or mistakenly, as constant, and therefore the same values are used for both physical and scaled models. This leads to the second partial scaling approach in which only the geometrical and kinematical related quantities are scaled properly, while all the material (mechanical and physical) properties, such as stiffness, Young's modulus, surface energy, surface tension and viscosity etc, are treated as constant and thus the actual values in the SI systems are used. Unsurprisingly, this apparently inconsistent approach will violate the scale-invariant property of 
some interaction laws and lead to some serious modelling issues. The full implications of this partial scaling approach will be addressed in the next section.

Remark 3 (Total computational costs) Assuming that the same time-stepping integration scheme will be adopted for solving both physical and scaled models, it is not difficult to deduce that the scale factor for the time step $\Delta t$ associated with the time-stepping scheme should be $\lambda_{\Delta t}=\lambda_{T}=h$, which implies that the same number of time steps are required in the scaled model as in the physical model. In other words, the same computational costs will be involved in solving both physical and scale models, and thus the exact scaling offers no computational cost savings. In order to reduce the computational costs for a large scale problem, some nonexact scaling approaches, such as coarse-graining, has to be employed, which will be the main theme of Part 2 of this paper.

\section{Scale invariance of interaction laws and pseudo-interaction laws}

Table 2 indicates that both particle strain and stress, as defined in Sect. 2.2, remain the same for any scale factor $h$, i.e. they are scale invariant. Since the particle strain and stress are related to the overlap (or other contact related geometrical quantities) and the contact force which are defined by interaction laws, all the interaction laws must therefore also satisfy certain conditions. More specifically, consider the general stress-strain form of an interaction law $\sigma=\sigma(\epsilon, R)$. Since the particle strain $\epsilon$ is dimensionless and independent of the scale factor $h$ to ensure that the particle stress $\sigma$ is also independent of the scale factor, the function $\sigma(\epsilon, R)$ must be dependent only on the strain and be independent of the particle intrinsic length $R$ :

$\sigma(\epsilon, R)=\sigma(\epsilon)$

An interaction law satisfying the above condition is termed scale-invariant. As discussed in the preceding section, all interaction laws will be scale-invariant if all the quantities involved in the model are scaled according to the scaling conditions that have been established. However, when all the material properties in interaction laws are viewed as constant, some interaction laws will violate the scale-invariant property.

\subsection{Scale-invariant check for interaction laws}

Now the scale-invariant property of some commonly used interaction laws in discrete element modelling will be examined under the condition that all physical parameters are constant. Those interaction laws that are found to be scale- invariant can be safely employed in a scaled model without any modification, while this is not the case for those laws that are not scale-invariant.

(1) Linear (spring) contact law $F=k u$. This is the most commonly used (normal) contact law where $k$ is the (normal) stiffness coefficient. The corresponding particle strain-stress relation has different forms for $2 \mathrm{D}$ and $3 \mathrm{D}$ cases as follows:

$\sigma(\varepsilon, R)= \begin{cases}k \varepsilon & \text { for } 2 \mathrm{D} \\ k \varepsilon / L & \text { for } 3 \mathrm{D}\end{cases}$

It is clear that if the coefficient $k$ is assumed to be constant, the linear contact law is scale-invariant for $2 \mathrm{D}$ problems but not for 3D. In other words, when the linear contact law is employed in a scaled 3D model, the coefficient $k$ must not be a constant but be related to the particle radius $R$.

(2) The Hertzian contact law (for $3 D$ ). The normal contact force between two linear elastic spheres of radii $R_{1}$ and $R_{2}$ is given by [14]

$F=\frac{4 E^{*}}{3}\left(R^{*} u^{3}\right)^{1 / 2}$

where $R^{*}$ is the effective radius:

$\frac{1}{R^{*}}=\frac{1}{R_{1}}+\frac{1}{R_{2}}$

and $E^{*}$ is the effective Young modulus:

$\frac{1}{E^{*}}=\frac{1-v_{1}^{2}}{E_{1}}+\frac{1-v_{2}^{2}}{E_{2}}$

in which $E_{1}, E_{2}$ are the elastic moduli and $\nu_{1}, v_{2}$ the Poisson's ratios associated with each body. The corresponding strain-stress form is

$\sigma(\varepsilon, R)=\frac{4 E^{*}}{3} \varepsilon^{3 / 2}$

where the characteristic length is taken to be $L=R^{*}$. Clearly, the form is particle size independent. Thus it is concluded that the Hertzian contact law is scale-invariant for 3D spherical particles.

(3) Coulomb friction models. The classic Coulomb model states that the friction force $\mathbf{F}_{t}$ can be calculated as

$\mathbf{F}_{t}=-\mu F_{n} \dot{\mathbf{u}}_{t} /\left\|\dot{\mathbf{u}}_{t}\right\|$

where $\mu$ is the coefficient of friction, $F_{n}$ is the magnitude of the normal contact force and $\dot{\mathbf{u}}_{t}$ is the tangential contact velocity. Obviously, this friction model will be scaleinvariant if $F_{n}$ is scale independent.

A regularised version of the classic Coulomb model (Fig. 2) is, however, often adopted in DEM [8]:

$\mathbf{F}_{t}=-\frac{\dot{\mathbf{u}}_{t}}{\left\|\dot{\mathbf{u}}_{t}\right\|} \begin{cases}k_{t} u_{t} ; & k_{t} u_{t}<\mu F_{n} \\ \mu F_{n} ; & k_{t} u_{t} \geq \mu F_{n}\end{cases}$ 


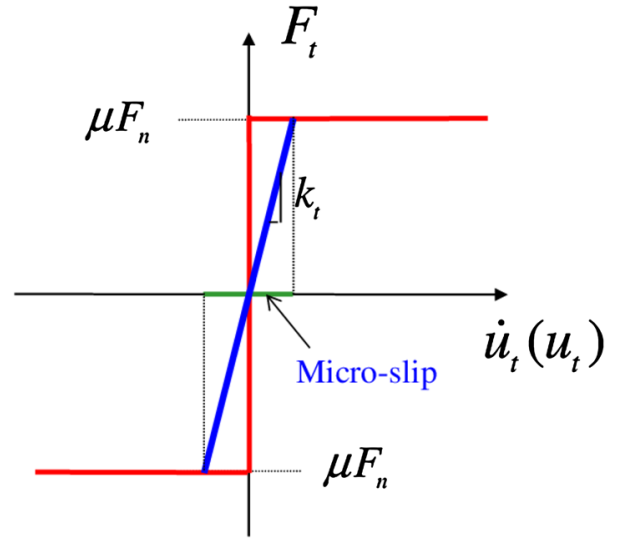

Fig. 2 Classic and regularised Coulomb friction models

where $u_{t}$ is the accumulated tangential displacement or sliding distance, and $k_{t}$ is the tangential stiffness coefficient which is normally a constant. Following the same argument as in the linear contact law above, it can be concluded that the regularised Coulomb model is scale-invariant for 2D cases but not for 3D cases.

(4) Viscous damping model. A damping force or a similar mechanism needs to be included in the DEM formulation to account for energy dissipation in particle systems. A viscous damping model is most commonly used for both local and global damping, in which the damping force is defined as

$F_{d}=2 m \omega \xi v$

where $m$ is the (contact or effective) mass; $\omega$ is the natural frequency (if properly defined); and $\xi$ is the damping ratio, a non-dimensional constant. It is easy to verify based on Table 2 that the viscous damping model is scale-invariant.

(5) Contact models for adhesive elastic particles. There are two commonly used contact models for two adhesive contacting spherical particles: The JKR and DMT models [14-17]. The former is more suitable for soft particles, while the latter is more appropriate for hard particles. The JKR model states that the normal contact force between two contacting adhesive elastic spheres can be expressed as (see also Fig. 3):

$F_{n}=\frac{4 E^{*} a^{3}}{3 R^{*}}-\sqrt{8 \pi \Delta \gamma E^{*} a^{3}}$

where $a$ is the contact radius; $\Delta \gamma=\gamma_{1}+\gamma_{2}-\gamma_{12}$ is the surface energy in which $\gamma_{1,2}$ are the adhesive surface energy per unit of area for both surfaces respectively, and $\gamma_{12}$ is the interfacial surface energy. The first term on the right hand side of the formula corresponds to the standard Hertzian contact force and the second term is the contribution from the particle adhesion. Converting the formula to the stress-strain form will show that the first term is scale-invariant as already established, but that the second term is not. Therefore, the

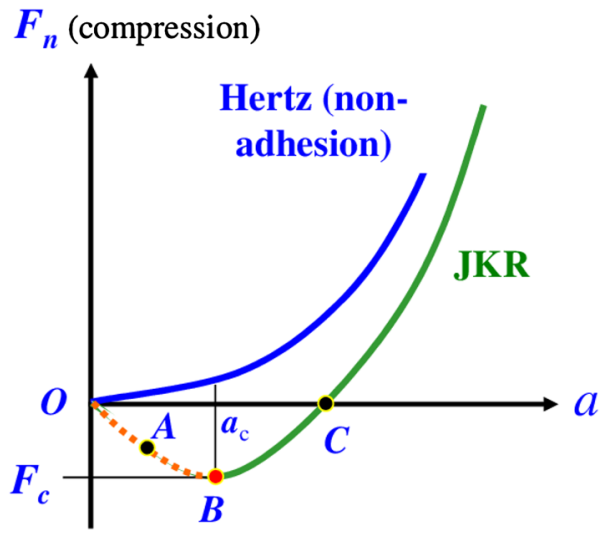

Fig. 3 Hertz and JKR models

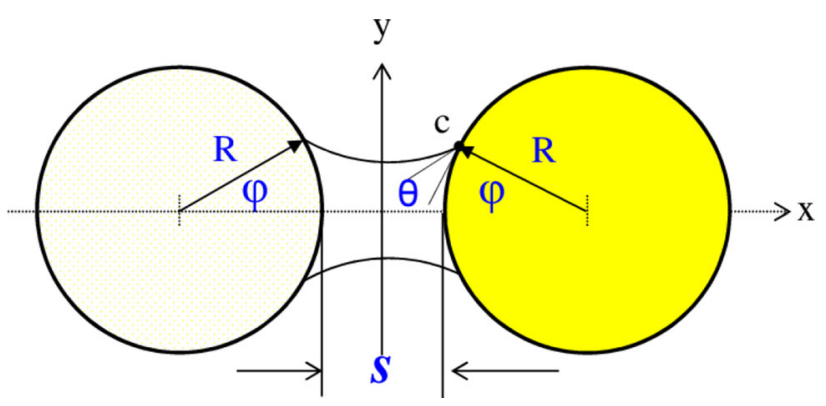

Fig. 4 Liquid bridge force between two wet spheres

JKR model is not scale-invariant. A similar conclusion can be drawn for the DMT model.

(6) Liquid bridge force between two wet spheres (3D). The cohesive force between two adjacent spheres (assuming the same size) due to the capillary effect is normally described by [18]

$$
F=\underbrace{2 \pi \kappa R \sin \phi \sin (\phi+\theta)}_{\text {surface tension }}+\underbrace{\pi R^{2} \Delta p(R, u, \phi, \theta) \sin ^{2} \phi}_{\text {hydrostatic pressure }}
$$

where $\kappa$ is the surface tension of the liquid; $\phi$ is the half filling angle; $\theta$ is the solid-liquid contact angle; and $\Delta p$ is the pressure difference across the air-liquid interface depending on $R, \phi, \theta$ and $u$ (which is now the separation distance of the two spheres, $s$ ) (see Fig. 4) and is normally determined by the Laplace-Young equation [20]. The corresponding strainstress form reads

$\sigma(\varepsilon, R)=\left[\pi \kappa \sin \phi \sin (\phi+\theta)+\frac{\pi}{4} \Delta p^{*}(\varepsilon, \phi, \theta) \sin ^{2} \phi\right] / L$

where $\Delta p^{*}$ is a dimensionless pressure independent of the particle size $R$ and dependent on the particle strain $\varepsilon$ and thus is scale-invariant. Nevertheless, the presence of the characteristic length $L$ in (26) indicates that the liquid bridge induced cohesive force is not scale-invariant in 3D cases. Again, this 
highlights a potential problem when a liquid bridge force model is used without modifications in a scaled model involving wet particles.

(7) Van der Waals forces between two spheres [19]. For modelling very fine particles, Van der Waals forces may play a very important role, and collectively represent weak intermolecular bonding (attractive) forces between two particles at a microscopic level. The so called Hamaker model is often applied in DEM for fine particle simulations, which states that the attractive force between two spheres is given by

$F=\frac{A R^{*}}{6 u^{2}}$

where $A$ is the Hamaker constant; $R^{*}$ is the equivalent radius of the two spheres, as defined in the Hertz model above; and $u$ is the separation distance of the two spheres. Converting it into the stress-strain form will reveal that the Hamaker modle is stress-strain form reads

$\sigma(\varepsilon, R)=\frac{A}{6 R^{* 3} \epsilon^{2}}$

(8) Hydro-dynamic forces acting on spheres. When a fluidparticle interaction problem such as a fluidised bed is simulated by coupling DEM and fluid solvers, the hydrodynamic forces acting on a particle from the fluid flow may need to be determined by using some empirical formula. The drag force acting on a sphere in a fluid flow, for instance, can be expressed as

$F_{d}=\frac{1}{2} \rho_{f} v^{2} c_{d} A$

where $\rho_{f}$ is the mass density of the fluid; $v$ is the relative speed of the sphere to the fluid; $A$ is the cross-section area of the sphere; and $c_{d}$ is called the drag coefficient. Clearly the scalability of this drag force equation entirely depends on the corresponding features of $c_{d}$. There are many empirical formulae available for $c_{d}$ and only the well-known Ergun Eq. [21] for a sphere in a packed particle bed is discussed below:

$c_{d}=\frac{1-\varepsilon}{2 \varepsilon^{3}}\left(150 \frac{1-\varepsilon}{\operatorname{Re}_{p}}+1.75\right)$

where $\varepsilon$ is the void fraction of the solid particles, and $\operatorname{Re}_{p}$ is the particle Reynolds number defined as

$\operatorname{Re}_{p}=\frac{d v}{v}$

in which $d$ is the diameter of the sphere and $v$ is the kinematic viscosity of the fluid. Because $\operatorname{Re}_{p}$ is proportional to the particle diameter, and the speed $v$ is scale-invariant, it can be concluded that the Ergun coefficient $c_{d}$ is not scale-invariant and thus the corresponding drag force Eq. (29) is also not scale-invariant.

The scalability of additional particular interaction laws can also be assessed in a similar fashion.

\subsection{Pseudo-particles and pseudo-interaction laws}

The scale-invariance assessment conducted in the preceding subsection reveals that most of commonly used interaction laws are not scale-invariant which is required for an exact scaling when all the material properties in interaction laws are viewed as constant. This means that when such interaction laws are used in their current forms in a scaled DEM model, the results obtained from the model cannot be properly scaled back to the physical model, and therefore may be incorrect. This is a serious problem that has not been well recognised by some DEM practitioners. The problem can be overcome, however, by introducing so called pseudoparticles and pseudo-interaction laws.

The particles in a physical problem are real, while the particles used in the scaled DEM model are not, and thus can be called pseudo-particles. A non-scale-invariant interaction law means that it is valid only for the actual sized particle in the physical model. Consequently, when such an interaction law is applied to a scaled or pseudo particle, the interaction law has to be modified such that the resulting pseudo form satisfies the scale-invariant requirement. To meet this requirement, the scale factor $h$ has to be present in the modified form. An interaction law that has undergone such a modification is thus termed the pseudo- or scaled interaction law and can be written in a general form as

$\overline{\mathbf{F}}=\overline{\mathbf{F}}(\bar{u}, \bar{R}, h)$

where $\overline{\mathbf{F}}, \bar{u}$ and $\bar{R}$ are the force, displacement and size of the particle in the scaled model respectively. A scaled interaction law should reduce to its original form when $h=1$. In some cases, it may be more convenient to use the actual size of a physical particle as a reference size, together with the scale factor, in the scaled interaction law. A few examples are given below to illustrate this scaling procedure.

First consider the linear contact model $F=k u$ which is not scale-invariant for 3D cases as established earlier. This means that $k$ has to be considered as the one which is particularly chosen only for the physical problem concerned, and therefore the same value cannot be used for a scaled model. However, if $k$ is also scaled to be $h k$, it gives rise to the following scaled linear contact law which is now scale-invariant:

$\bar{F}=h k \bar{u}$

The liquid bridge model (26) is taken as the second example. The scaled model can be written as

$$
\begin{aligned}
\bar{F}= & {[2 \pi \gamma \bar{R} \sin \phi \sin (\phi+\theta)} \\
& \left.+\pi \bar{R}^{2} \Delta p(\bar{R}, \bar{u}, \phi, \theta) \sin ^{2} \phi\right] h
\end{aligned}
$$

which is obtained by multiplying the original form by the scale factor $h$. Similarly, the Hamaker model (27) should be scaled to 
$\bar{F}=\frac{h^{2} A \bar{R}^{*}}{6 \bar{u}^{2}}$

For hydrodynamic force calculations, the particle Reynolds number defined by Eq. (31) should remain the same after scaling:

$R e_{p}=\frac{\bar{d} v}{h v}$

Other non-scale-invariant interaction laws can also be properly scaled to become scale-invariant in a similar fashion.

Note that the same modified form of an interaction law can be obtained directly from the original form by applying the scaling conditions in Table 2 to all the material parameters involved but not to the geometrical or kinematic quantities.

\section{Concluding remarks}

The present work has developed a simple but powerful and generic theoretical framework for establishing a comprehensive set of scale factors under which a scaled discrete element model can exactly reproduce the mechanical behaviour of a physical model. The whole framework is based on directly dealing with the governing equations for general particle systems. In particular, three basic physical quantities, different from the conventional SI system, can be freely chosen. In addition, the scale factors of these three basic quantities between both physical and scaled models can also be arbitrarily set. As addressed earlier, different selections of three basic quantities and their corresponding scale factors will lead to different sets of scale factors. This offers a possibility to develop the most appropriate scaling model for a given particle problem. Although the development is for mechanical systems only, other physical phases such as thermal, magnetic and electrical can be readily included and tackled in a similar fashion.

A particular set of three basic quantities has been chosen in which mass density replaces mass to be a basic quantity, and the three basic scale factors are also specially tuned to derive a unique set of scale factors for the physical quantities encountered in discrete element modelling of particle systems. The direct consequence of such a selection is that the artificially defined particle stress and strain must be independent of the spatial scale factor $h$, which further leads to the conclusion that all the interaction laws to be used in a scaled model must be scale-invariant. The subsequent examination reveals that, if all material (mechanical and physical) properties are treated as constant, most commonly used interaction laws do not possess such a feature essential to achieve an exact scaling, and therefore cannot be directly used in a scaled model. This problem has been overcome by treating the scaled particles as pseudo-particles and by properly scaling the interaction laws. The resulting scaled interaction laws become scale-invariant and thus can be employed in a scaled model.

It is worth mentioning that the scale-invariance of the particle stress and strain and the subsequent scale-invariant requirement for all the interaction laws are not unique to our particular choice of three basic quantities and their scale factors, but can also be obtained from other selections. For instance, if force $F$ is chosen to be a basic quantity instead of density $\rho$, and the corresponding scale factor is set to be $\lambda_{F}=h^{2}$, the same scaling conditions as listed in Table 2 and thus the same scaling laws can be retained. In fact, the scaleinvariance must serve as a necessary condition for achieving an exact scaling in all cases.

Nevertheless, it is also revealed that no computational gains can be obtained from an exact scaling. Basically, enforcing all the exact scaling conditions is computationally equivalent to the modelling of the original physical problem without any scaling. Consequently, techniques such as coarse-graining have to be utilised to reduce the scale number $S_{n}$ of the discrete element model and thereby reducing the whole computer costs to a reasonable level. The next part of this three-part paper will address some fundamental issues associated with the coarse-graining technique.

\section{References}

1. Cundall PA, Strack ODL (1979) A discrete numerical model for granular assemblies. Geotechnique 29(1):47-65

2. Owen DRJ, Feng YT, Wang F, Cottrell MG, Pires FA, Yu J (2004) The modeling of multi-fracturing solids and particulate media. Int J Numer Methods Eng 60(1):317-340

3. Weinhart T, Hartkamp R, Thornton AR, Luding S (2013) Coarsegrained local and objective continuum description of threedimensional granular flows down an inclined surface. Phys Fluids 25(7):070605

4. Bierwisch C (2009) Numerical simulations of granular flow and filling. PhD thesis, University of Freiburg, Germany

5. Kuwagi K, Takeda H, Horio M (2004) The similar particle assembly (SPA) model: an approach to large-scale discrete element (DEM) simulation. In: proceedings of the international conference on fluidization engineering XI, Ischia, Naples, 243250

6. Sakai M, Koshizuka S (2009) Large-scale discrete element modeling in pneumatic conveying. Chem Eng Sci 64:533-539

7. Feng YT, Han K, Owen DRJ, Loughran J (2009) On upscaling of discrete element models: similarity principles. Eng Comput 26(6):599-609

8. Han K, Perić D, Crook AJL, Owen DRJ (2000) A combined finite/discrete element simulation of shot peening process. Part I: studies on 2D interaction laws. Eng Comput 17(5):593-619

9. Han K, Perić D, Owen DRJ, Yu J (2000) A combined finite/discrete element simulation of shot peening process. Part II: 3D interaction laws. Eng Comput 17(6/7):683-702

10. Kruyt NP (2003) Statics and kinematics of discrete Cosserat-type granular materials. Int J Solids Struct 40:511534

11. Douglas JF, Gasiorek JM, Swaffield JA, Jack LB (2005) Fluid Mech, 5th edn. Pearson Education Ltd, London

12. Wood DM (2004) Geothechical modelling. Spon Press, London

13. Poschel T, Saluena C, Schwager T (2001) Scaling properties of granular materials. Phys Rev E 64:011308 
14. Johnson KL (1985) Contact mechanics. Cambridge University Press, Cambridge

15. Johnson KL, Kendall K, Roberts AD (1971) Surface energy and the contact of elastic solids. Proc R Soc Lond A 324:301-313

16. Derjaguin BV, Muller VM, Toporov YP (1975) Effect of contact deformations on the adhesion of particles. J Colloid Interface Sci 53(2):314-326

17. Muller VM, Derjaguin BV, Toporov YP (1983) On two methods of calculation of the force of sticking of an elastic sphere to a rigid plane. Colloids Surf 7(3):251-259
18. Lian GP (1994) Computer simulation of moist agglomerate collisions. PhD thesis, the University of Aston in Brimingham, UK

19. Yang RY, Zou RP, Yu AB (2000) Computer simulation of the packing of fine particles. Phys Rev E 62(3):3900-3908

20. Lamb HS (1928) Including hydrostatics and the elements of the theory of elasticity, 3rd edn. Cambridge University Press, Cambridge

21. Ergun S, Orning AA (1952) Fluid flow through packed columns. Chem Eng Prog 48:89-94 